\title{
Propiedades Psicométricas del Test de Ajuste Psicológico a la Separación en personas separadas y divorciadas chilenas
}

\section{Psychometric Properties of the Psychological Adjustment to Separation Test in Divorced and Separated Chileans}

\author{
Mónica Guzmán-González \\ María Alejandra Barraza \\ Francisca Bastías \\ Damaris Bustamante \\ María Fernanda Ojeda \\ Escuela de Psicología, Universidad Católica del Norte, Chile
}

Recibido (1 de octubre de 2017) Aceptado (20 de junio de 2018)

\begin{abstract}
Resumen
La ausencia de instrumentos adaptados para la medición del ajuste al divorcio y la separación en Chile representa una barrera para el desarrollo de estudios e intervenciones que aborden el tema del divorcio en este contexto particular. El objetivo de este estudio fue evaluar la validez y fiabilidad de las puntuaciones del Test de Adaptación Psicológica a la Separación (PAST), cuestionario de autoinforme dividido en dos partes independientes que evalúan por un lado el apego a la pareja y la soledad negativa (PAST-A) y, por otro, la coparentalidad negativa (PAST-B). Para ello, se evaluaron dos muestras independientes de personas separadas y divorciadas chilenas $(\mathrm{N}=141 ; \mathrm{N}=594)$. De los resultados obtenidos, se puede concluir que el PAST posee propiedades psicométricas adecuadas en términos de su fiabilidad y validez. Se discuten las implicancias clínicas de estos hallazgos.

Palabras clave: Divorcio, separación, adaptación al divorcio y la separación.
\end{abstract}

\begin{abstract}
The lack of instruments adapted to the Chilean context to measure the adjustment to divorce and separation represents an important constraint to the development of a line of research and intervention focused on the topic of divorce in this particular context. The objective of this study was to assess the psychometric properties of the Psychological Adjustment to Separation Test (PAST) Chilean version, which is divided into two parts that evaluate former partner attachment and lonely negativity (PAST-A) and coparenting conflict (PAST-B). To that end, two independent samples of separated individuals $(\mathrm{N}=141 ; \mathrm{N}=594)$ were evaluated. From the results obtained, we concluded that the PAST has adequate psychometric properties for its use into the Chilean context. Clinical implications of these findings are discussed.
\end{abstract}

Keywords: Divorce, separation, adjustment to divorce and separation

Correspondencia: Mónica Guzmán-González, Escuela de Psicología, Universidad Católica del Norte, Chile. Email: moguzman@ucn.cl Correspondence: Mónica Guzmán-González, Escuela de Psicología, Universidad Católica del Norte, Chile. Email: moguzman@ ucn.cl Esta investigación ha sido financiada por el Fondo Nacional de Ciencia y Tecnología del Gobierno de Chile (Fondecyt 1150044), concedido a la primera autora. 


\section{Introducción}

El divorcio y la separación, entendidos ambos como la disolución del eje pareja, constituyen procesos complejos, cuyos efectos si bien están sujetos a una amplia variabilidad, suelen asociarse a altos niveles de estrés, de carácter más o menos transitorio, que impactan la salud mental, así como el bienestar subjetivo de las personas (Amato, 2010, Sbarra, 2015). Se ha reportado por ejemplo que, como grupo, las personas divorciadas experimentan menores índices de bienestar y más problemas de salud mental en comparación a la personas casadas (Afifi, Cox \& Enns, 2006; Amato, 2010; Evans \& Kelley, 2004; Hughes \& Waite, 2009; Sbarra, 2006; Wang \& Amato, 2000). Además, existen antecedentes que documentan que las personas divorciadas están menos satisfechas con ciertos aspectos de su vida, como aquellos asociados a las relaciones familiares y a sus redes sociales, que las personas casadas (Forste \& Heaton, 2004).

El estrés asociado al divorcio y la separación guarda relación con que la disolución de la pareja implica una serie de cambios y demandas de acomodación en diferentes esferas, como la familiar, económica y social (Cáceres, Manhey \& Raies, 2004). Al mismo tiempo, involucra un proceso psicológico de adaptación al nuevo estatus, denominado ajuste al divorcio y la separación (ADS; Amato, 2000; Gähler, 2006; Evans \& Kelley, 2004).

Una de las propuestas de conceptualización del ADS es la elaborada por Sweeper \& Halford (2006), quienes a partir de una revisión de la literatura sobre el tópico, concluyeron que el ADS implica tres desafíos específicos. El primero y el más evidente, es adaptarse a la pérdida de la pareja, que si bien en algunos casos puede producir alivio, en otros se manifiesta en un anhelo permanente de cercanía emocional y en la persistencia de sentimientos amorosos hacia el o la ex cónyuge, aspecto que los autores denominan apego a la ex pareja. El segundo desafío es el logro de un sentido de balance afectivo e integración social, dado que la pérdida de las redes sociales asociadas a la ex pareja, junto a la ruptura misma, puede generar sentimientos de soledad y aislamiento, así como mayor labilidad emocional (Wang \& Amato, 2000). El último desafío, en aquellos casos en que ha habido hijos en común, guarda relación con que ambos padres logren coordinarse para ejercer una coparentalidad positiva, tarea que puede ser compleja cuando el divorcio y la separación han estado caracterizados por altos niveles de conflicto.

Pese a que el tema del ADS desde una perspectiva psicológica y clínica ha sido ampliamente investigado en el concierto internacional, la proporción de estudios empíricos realizados en contexto latinoamericano son escasos y en Chile, en particular, casi inexistentes. Tal carencia, limita la posibilidad de comprender un fenómeno que no es in- dependiente del contexto sociocultural en que está inserto. Ello porque es posible que el sistema de creencias sociales acerca del divorcio en particular, así como el valor cultural asignado al matrimonio como institución, afecte la manera en que las personas afrontan una ruptura. Chile, además, tiene la particularidad de ser uno de los últimos países a nivel mundial en haber legalizado el divorcio, el año 2004.

Una de las principales limitantes para el desarrollo de una línea de investigación acerca de las consecuencias del divorcio y la separación desde una perspectiva psicológica

es la falta de instrumentos creados o adaptados en el contexto chileno, que permitan medirel ADS, teniendo como foco el adulto que se separa.

\section{Medición del Ajuste al Divorcio (ADS)}

En relación con la medición del ADS se ha tendido a utilizar una aproximación multidimensional, con diversos indicadores de ajuste general como la salud mental, el bienestar subjetivo, la afectividad positiva, entre otros. Sin embargo, una debilidad de estas medidas generales de ajuste es que resulta complejo establecer si la separación o divorcio en sí misma conlleva detrimentos en la salud mental, o bien, las personas que ya poseen problemas de salud mental se encuentran en mayor riesgo de separarse (Amato, 2010). Por esto, resulta relevante contar con instrumentos para evaluar de manera específica el ADS, dado que la adaptación a este nuevo estatus de vida supone desafíos particulares que no son capturados completamente por medidas generales de ajuste (Sweeper \& Halford, 2006; Yárnoz-Yaben \& Comino, 2010).

Entre los instrumentos disponibles, uno de los más conocidos es la Fisher Divorce Adjustment Scale (FDAS, Fisher, 1978), que evalúa una serie de sentimientos y experiencias pertinentes exclusivamente a las personas divorciadas (Yilmaz \& Fisiloglu, 2006) en 6 dimensiones: desapego de la relación, sentimientos de rabia, autoestima, síntomas de duelo, reconstrucción de las relaciones sociales y autoaceptación social. Desarrollado como un instrumento utilizado en el contexto del trabajo terapéutico grupal con personas divorciadas, una de sus críticas es que su estructura factorial no estaba del todo establecida (e.g. Kellas \& Manusov, 2003; Yilmaz \& Fisiloglu, 2006) y que su excesiva extensión, dificulta su aplicación en contextos masivos. En la actualidad, ya se cuenta con la validación reciente al contexto chileno de esta escala en una versión breve (Guzmán, Garrido, Calderón, Contreras \& Rivera, 2017).

Otro instrumento utilizado es el Cuestionario de Adaptación al Divorcio-Separación (CAD-S, Yárnoz-Yaben, 2010). Este último posee una alta consistencia interna y una adecuada validez de constructo, demostrada por la 
relación de esta escala con medidas de satisfacción vital en los progenitores y con problemas conductuales en los niños. Esta escala es particularmente aplicable a personas divorciadas con hijos en común menores de 18 años, dado su énfasis en aspectos vinculados a la coparentalidad (Yárnoz \& Comino, 2010).

Adicionalmente, se cuenta con el Psychological Adjustment to Separation Test (PAST, Sweeper \& Halford, 2006), objeto del presente estudio, que está dividido en dos partes independientes la parte A que evalúa las dimensiones antes descritas de apego a la ex pareja y soledad negativa y la parte $\mathrm{B}$, que evalúa la coparentalidad negativa y que se aplica a aquellas personas separadas que tienen hijos en común menores de 18 años. Considerando que el foco de este instrumento está puesto sobre el ajuste psicológico, éste fue desarrollado para su uso con personas separadas provenientes tanto de uniones legales como de hecho.

Hasta donde se tiene conocimiento, se cuenta con la validación del PAST a población portuguesa (Lamela, Figuereido, Bustos \& Martins, 2014), una adaptación al alemán (Smet, Buysse \& Brondeel, 2011) y al castellano con población mexicana (Rodriguez, 2011; Rodríguez \& Ribeiro, 2013). En la versión portuguesa, en la que se evaluaron las propiedades psicométricas exclusivamente de la parte A, se replicó la estructura factorial propuesta por los autores, y se aportó evidencia de su consistencia interna, así como de su validez convergente, divergente y discriminante.

Por su parte, en la versión alemana, sólo se hizo un proceso de traducción cruzada, reportándose únicamente antecedentes acerca de su consistencia interna, pero no de su validez (Smet et al., 2011).

Adicionalmente, se dispone de la adaptación del PAST en población mexicana desarrollada por Rodríguez (2011), siendo esta versión la que se ocupó como base para el proceso de validación en contexto chileno. En dicho estudio se realizó un proceso de traducción cruzada y luego se examinó su estructura factorial, identificándose una tercera dimensión a la que denominó inestabilidad emocional; los índices de fiabilidad fueron adecuados. La coparentalidad negativa no fue tomada en cuenta, debido a que el autor puso su foco atención sólo en lo que ocurre con las personas separadas y no en su rol de padres.

Pese a que la escala ya se encuentra traducida al español, las diferencias culturales y lingüísticas que pueden detectarse aun cuando el idioma sea el mismo, fundamentan la necesidad de validar elPAST en contexto chileno, siendo además parte de las recomendaciones señaladas por Hambleton, Merenda \& Spielberg (2005) sobre el uso de instrumentos en contextos diferentes para el cual fueron diseñados.

De esta manera, el objetivo de nuestro estudio fue evaluar la validez y confiabilidad de las puntuaciones del Psy- chological Adjustment to Separation Test (PAST, Sweeper \& Halford, 2006) para su uso en personas divorciadas y separadas chilenas. Específicamente, examinamos la estructura factorial del instrumento, así como la invarianza métrica de la escala según sexo y tipo de relación (matrimonios/ uniones de hecho); asimismo, estimamos su consistencia interna y finalmente los examinamos su asociación con constructos afines, pero diferentes, tales como la salud mental y la satisfacción con la vida, dados antecedentes previos que documentan una asociación directa entre problemas en el ADS y la salud mental e inversa con la satisfacción con la vida.

\section{Método}

\section{Participantes}

Muestra 1. Se evaluó una muestra piloto compuesta por 141 personas separadas y divorciadas. La distribución por género fue de 78 mujeres y 63 hombres, con un promedio de edad de 39.85 años ( $\mathrm{DE}=14.03)$. Un 39.3\% de la muestra tenía hijos en común y un $44.2 \%$ provenía de relaciones de convivencia, mientras que el $55.8 \%$ de matrimonios. El tiempo promedio de duración de la relación fue de 7.43 años (DE=6.83) y la ruptura había ocurrido en promedio hace 2.95 años $(D E=1.95)$.

Muestra 2. Se evaluó una muestra no probabilística balanceada según sexo y edad, conformada por 594 personas divorciadas o separadas, provenientes de vínculos legales (matrimonios) y no legales (cohabitación). Se decidió incluir estos dos tipos de uniones por cuanto los procesos psicológicos desplegados ante una ruptura se darían en ambos tipos de relaciones, con independencia del estatus legal (Sweeper \& Halford, 2006).

Del total de participantes, 344 (57.9\%) fueron mujeres y $250(42.1 \%)$ hombres, con un promedio de edad de 40.27 años $(D E=11.46)$. En relación con el nivel educacional, un 73.3\% de la muestra tenía estudios superiores (universitarios o técnicos), un $23.7 \%$ enseñanza media, mientras que el 3 $\%$ poseía estudios inferiores a éstos. Un $45.1 \%$ de los participantes provenía de relaciones de convivencia, mientras que $54.9 \%$ correspondió a matrimonios. El tiempo promedio de duración de la relación con la ex pareja fue de 11.17 años $(D E=9.34)$ y se habían separado hace un promedio de 3.30 años $(D E=2.16)$. Se consideró el cese de convivencia como criterio para establecer el tiempo desde la separación. Un $73.4 \%$ reportó hijos en común con la ex pareja, y entre dichos participantes, 314 señalaron tener hijos menores de 18 años. 


\section{Instrumentos}

Psychological Adjustment to Separation Test (PAST, Sweeper \& Halford, 2006), adaptado lingüísticamente al castellano por Rodríguez (2011). Este instrumento es utilizado para medir el ajuste a la separación y/o divorcio y consta de tres subescalas separadas en dos partes que son aplicadas de manera separada. La parte A, compuesta de 19 ítems, está formada por las dos dimensiones descritas previamente: soledad negativa y el apego a la ex pareja. Ejemplos de ítems de la primera y segunda subescala son "me siento aislado/a" y "pienso constantemente en mi ex pareja" respectivamente. La Parte $\mathrm{B}$, compuesta de 7 ítems, se aplica en aquellas personas separadas y divorciadas que tengan hijos en común menores de 18 años y evalúa la coparentalidad negativa, es decir, la presencia de problemas en el ejercicio de la parentalidad (Ej: "mi ex pareja y yo evitamos hablarnos"). En conjunto, el PAST se compone de 26 ítems, medidos a través de Escala Likert de 5 puntos ( 1 , totalmente en desacuerdo y 5 , totalmente de acuerdo). Puntajes más altos indican mayores problemas de ADS.

Los resultados reportados por los autores (Sweeper \& Halford, 2006) avalan las buenas propiedades psicométricas del PAST, considerando sus antecedentes de fiabilidad y validez, para examinar las diferencias interindividuales en el ajuste al divorcio entre personas separadas y divorciadas. La consistencia interna de cada subescala fluctuó .90 y .89 para la soledad negativa y entre .88 y .89 para el apego a la ex pareja. En el caso de la coparentalidad, fiabilidad se ubicó entre .83 y .86. Además, la escala se relacionó con variables de personalidad medidas con el Eysenck Personality Questionnaire (Eysenck, Eysenck \& Barrett, 1985) y con salud mental (Lovibond \& Lovibond, 1995).

Esta escala fue el único instrumento aplicado a la muestra piloto. En la segunda muestra se aplicaron además los siguientes instrumentos.

The Abbreviated Scales of Depression, Anxiety and Stress (Lovibond \& Lovibond, 1995), en su versión validada en Chile por Vinet, Rehbein, Román \& Saiz (2008; DASS21). Este instrumento se utiliza para medir sintomatología depresiva, ansiosa y estrés. Está organizado en 21 ítemsen total, puntuados de 0 (no describe nada de lo que me pasóo sentí durante la semana) a 3 (sí, esto me pasó mucho, o casi siempre) en escala Likert. En el instrumento de Lovibond \& Lovibond (1995), la consistencia interna basada en el alfa de Cronbach fue de 0.91 en la escala de depresión, 0.84 en la escala de ansiedad y 0.90 en la escala de estrés. Para este estudio la confiabilidad fue de 0.88 .

Escala de Satisfacción con la Vida (Diener, Emmons, Larsen \& Griffin, 1985). validado en Chile por VeraVillarroel, Urzúa, Pavez, Celis-Atenas \& Silva(2012). Este instrumento mide el nivel de satisfacción vital en 5 ítems
(Ej: "las condiciones de mi vida son excelentes") a los que se responde a través de una Escala Likert de 7 puntos, (1, no, en absoluto y 7 sí, totalmente). El índice de confiabilidad interna es de 0.82 . Por otro lado, se obtuvo evidencia de su validez mediante su asociación con constructos teóricos relacionados. Para el presente estudio la confiabilidad fue de 0.81 .

\section{Procedimiento}

Este estudio contó con la aprobación del comité de ética de la Universidad Católica del Norte y del Fondo Nacional de Desarrollo Científico y Tecnológico(CONICYT).

La recolección de datos constó de 3 fases: en la primera se realizó la adaptación lingüística de los ítems del PAST al contexto chileno, ocupando como base la versión mexicana de Rodríguez (2011). Para ello, un equipo conformado por tres psicólogos clínicos, especialistas en temas de pareja, revisó independientemente cada uno de los reactivos del instrumento. Luego de ello, se reunieron y elaboraron una versión consensuada del mismo. Esta versión se aplicó a una muestra piloto, a fin de explorar la estructura factorial del instrumento. Posteriormente, se inició la etapa de recolección de datos con la muestra de validación. Para ello se conformó un equipo de encuestadores en cuatro ciudades de Chile, quienes utilizaron las redes sociales (tanto virtuales como directas), para contactar a posibles participantes que cumpliesen con los criterios de inclusión. A todos ellos se les entregó información sobre los objetivos y naturaleza del estudio, enfatizando la confidencialidad y el carácter voluntario de su participación en el mismo. La aplicación del instrumento fue en papel.

\section{Análisis de Datos}

De manera previa a los análisis, se examinaron los datos para evaluar el cumplimiento de los supuestos y detectar casos con elevados datos ausentes $(\mathrm{N}>10 \%)$, los que fueron removidos de la muestra. Para el examen de la estructura factorial del PAST-A se ocuparon dos estrategias. Primero se realizó un análisis paralelo (Horn, 1965) con la muestra piloto, a fin de determinar el número de factores, dados los antecedentes reportados por Rodríguez \& Ribeiro (2013) en muestra mexicana, en que se identificaron tres dimensiones. Para ello se ocupó el programa Factor 10.5 (Lorenzo-Seva \& Fernando, 2006).

Posteriormente, se llevó a cabo un Análisis Factorial Confirmatorio (AFC) con la segunda muestra. Los análisis se llevaron a cabo de manera separada para la Parte A y B, dado que ambas pueden ser usadas independientemente y es el modo en que la escala original fue analizada por los propios autores del instrumento (Sweeper \& Halford, 
2006). Además del estadístico chi cuadrado, se utilizaron los siguientes indicadores adicionales de ajuste: el RMSEA y su intervalo de confianza del 90\%, el Comparative Fit Index (CFI), el Tucker-Lewis Index (TLI), el Root Mean Square Error of Approximation (RMSEA) y el Standardized Root Mean Square Residual (SRMR). Se definieron como criterios de buen ajuste valores sobre .95 en el CFI y el TLI, cercanos o menores a .08 en el caso del RMSEA y menores a .06 el SRMR, de acuerdo a lo propuesto por (Bentler, 1990). El análisis factorial confirmatorio se realizó con el programa Mplus y Máxima Verosimilitud como método de estimación, ocupando el algoritmo FIML para el manejo de datos perdidos.

Con los reactivos de la parte A se realizó además un análisis de invarianza métrica por género y tipo de relación (matrimonio y convivencia). El tamaño de la muestra no fue suficiente para llevar a cabo el mismo análisis con la parte B sobre coparentalidad, dado que sólo fue respondida por aquellos participantes que tenían hijos en común menores de 18 años. La evaluación de la equivalencia del modelo de dos factores según género y tipo de relación se realizó mediante la comparación de modelos anidados, progresivamente más restrictivos. Para ello se evaluaron tres tipos de invarianza: configural, en la que el número de factores y los patrones de cargas factoriales se estimaron libremente para ambos grupos; invarianza métrica, en la que se restringió a ser iguales las cargas factoriales; e invarianza escalar o métrica fuerte, en la que además se restringieron a ser iguales los interceptos. Para evaluar si estos modelos progresivamente más restrictivos difieren entre sí, se ocuparon como criterios la diferencia en el chi cuadrado, así como los índices de ajuste comparativo propuestos por Millsap \& Cham (2012) \& Chen (2007): valores menores a .010 en el CFI, a .05 en el TLI y de .001 en el RMSEA se consideran como indicadores de equivalencia de los modelos.

Finalmente, se estimó la fiabilidad para cada una de las subescalas mediante el cálculo del alfa ordinal y se aportó evidencia adicional de la validez de las puntuaciones del PAST mediante el examen de su asociación con variables teóricamente relacionadas, como la satisfacción con la vida y la salud mental.

\section{Resultados}

\section{Análisis Factorial Exploratorio del PAST-A}

Los resultados del análisis paralelo indican que el autovalor empírico del primer factor real (37.3) se encuentra por encima del promedio (11.2) y del percentil 95 de los autovalores aleatorios (12.3). Respecto del segundo factor, se identifica que el autovalor real (11.0) se encuentra por encima del promedio (10.2), y posee valor similar al del percentil 95 (11.0). A partir del tercer factor, los autovalores empíricos se encuentran por debajo de la media y del percentil 95 . Considerando estos resultados y ocupando el criterio más robusto, la solución más adecuada sería de un único factor; sin embargo, dado que el valor obtenido por el segundo autovalor real es igual al del percentil $95 \mathrm{y}$ hay antecedentes previos que avalan una solución de dos factores (Sweeper \& Halford, 2006; Lamela et al., 2014), optamos por una solución de dos factores como la más apropiada.

\section{Análisis Factorial Confirmatorio del PAST-A}

Estimamos un primer modelo, con los dos factores latentes originales identificados por los autores de la escala y 19 variables observadas. El modelo inicial evidenció un pobre ajuste a los datos, $\chi 2(151, N=594)=777.94, p<0.001$, $\mathrm{CFI}=0.895, \mathrm{TLI}=0.881, \mathrm{RMSEA}=0.084,90 \%$ IC $[.078$, .089]; SRMR $=.056$.

Al examinar las cargas factoriales se observa que hay dos ítems sin cargas significativas (reactivos 6,13 y 14) y que el ítem 1 tiene carga en ambos factores. Además, al observar los índices de modificación sugeridos por el programa, se identificó la presencia de dos pares de errores correlacionados de magnitud considerable, entre los ítems 13 y 19 (IM=116.070) y entre el reactivo 17 y 19 (IM=55.963).

Con base en estos resultados procedimos a re-estimar un segundo modelo, excluyendo los 4 ítems problemáticos, el cual evidenció un mejor ajuste que el anterior, aunque todavía por debajo de los valores considerados aceptables, $\chi^{2}(89, \mathrm{~N}=594)=378.125, p<0.001, \mathrm{CFI}=0.942, \mathrm{TLI}=0.931$, RMSEA $=0.074,90 \%$ IC [.066, .082]; SRMR $=.046$. Nuevamente, la inspección de los índices de modificación revelan la existencia de una correlación entre los errores de los ítems 17 y 19 (IM = 71.134).

Posteriormente, decidimos examinar un tercer modelo, en el que se permitiese la correlación entre los errores de los ítems 17 y 19, según la información aportada por los índices de modificación, dado que la revisión de ambos da cuenta de un traslape en sus contenidos.

$\mathrm{El}$ ajuste de este tercer modelo fue adecuado, $\chi 2(88, \mathrm{~N}=$ 594) $=306.401, p<0.001, \mathrm{CFI}=0.956, \mathrm{TLI}=0.947, \mathrm{RMSEA}$ $=0.065,90 \%$ IC $[.057, .073] ;$ SRMR $=.042$. El examen de los pesos factoriales muestra que todos los ítems tienen cargas significativas y de magnitud apropiada (>.30) y los factores estuvieron fuertemente correlacionados $(\mathrm{r}=.763)$ (ver tabla 2).

$\mathrm{Al}$ examinar mediante la diferencia en el $\chi^{2}$ para modelos anidados $\left(\Delta_{\chi_{2}}=\chi^{2}{ }_{\text {anidado }}-\chi_{\text {base }}^{2}\right)$, entre el modelo 2 y 3 , se observa que la diferencia entre ambos modelos, $\Delta_{\gamma 2}=$ $71.724, \Delta_{g l}=1$ es estadísticamente significativa, a un nivel de $p<.001$, por lo que el modelo 3 reproduce mejor la matriz 
Tabla 1. Resumen Índices de Ajuste de los Modelos Evaluados

\begin{tabular}{llllllll}
\hline MODEL & $\chi^{2}$ & $\mathrm{gl}$ & $\mathrm{CFI}$ & $\mathrm{TLI}$ & RMSEA & IC 90\% & \multicolumn{2}{c}{ SRMR } \\
\hline Modelo 1 & $777.937^{*}$ & 151 & .895 & .881 & .084 & $.078-.089$ & .056 \\
Modelo 2 & $378.125^{*}$ & 89 & .942 & .931 & .074 & $.066-.082$ & .046 \\
Modelo 3 & $306.401^{*}$ & 88 & .956 & .947 & .065 & $.057-.073$ & .042 \\
Modelo 4 & $1313.210^{*}$ & 152 & .806 & .782 & .066 & $.108-.119$ & .066 \\
\hline
\end{tabular}

Note. Modelo 1: Original con 19 ítems; Modelo 2: Modelo con 15 ítems (sin los reactivos 1, 6, 13 y 14, sin errores correlacionados); Modelo 3: Modelo con 15 ítems y una covarianza de error; Modelo 4: Unifactorial. $* p<.001$

de datos que el modelo 2 .

Finalmente, como forma de evaluar el ajuste de modelos alternativos al original y considerando la alta correlación entre las subescalas del PAST-A probamos una solución alternativa de un modelo unifactorial, el cual evidenció un pobre ajuste a los datos (ver tabla 1).

Considerando estos resultados, decidimos retener el modelo 3 como el que mejor representa la estructura de los datos observados. En la tabla 2 se presentan las cargas factoriales estandarizadas obtenidas en el PAST-A con el AFC.

Tabla 2. Ponderaciones de factores para el análisis factorial confirmatorio de las Subescalas del PAST

\begin{tabular}{lll}
\hline & Factor 1 & Factor 2 \\
\hline Item 2 & .830 & \\
Item 4 & .786 & \\
Item 5 & .865 & \\
Item 7 & .701 & \\
Item 8 & .590 & \\
Item 10 & .786 & \\
Item 18 & .501 & \\
Item 3 & & .784 \\
Item 9 & & .796 \\
Item 11 & & .367 \\
Item 12 & & .752 \\
Item 15 & & .809 \\
Item 16 & & .849 \\
Item 17 & .687 \\
Item 19 & & .623 \\
\hline
\end{tabular}

$\mathrm{N}=594$

\section{Análisis Factorial Confirmatorio Parte B}

Para la evaluación de la estructura factorial de la segunda parte de la escala, orientada a evaluar la coparentalidad negativa, ocupamos los mismos índices de ajuste previamente señalados. El modelo unifactorial propuesto por los autores evidenció un pobre ajuste a los datos, $\chi 2(14$, $N=314)=213.853, p<0,001, \mathrm{CFI}=0.754, \mathrm{TLI}=0.632$, RMSEA $=0.213,90 \%$ IC $[.189, .239] ;$ SRMR $=.130 . \quad$ La inspección de las cargas factoriales revela que los ítems 3 , 4 y 6 , es decir, aquellos que evalúan de manera directa las dificultades en la coparentalidad, tienen cargas inferiores a .30. Además, los índices de modificación sugeridos por el programa dan cuenta de la existencia de tres covarianzas de error $(\mathrm{IM}=30.066$ a 111.596) de gran magnitud entre dichos reactivos, antecedentes que sugieren la presencia de un segundo factor.

Considerando lo anterior, estimamos un segundo modelo, esta vez especificando dos factores, uno que agrupa los reactivos 1, 2, 5 y 7 y un segundo que contiene los ítems 3 , 4 y 6 , cuyo ajuste fue satisfactorio, $\chi 2(13, \mathrm{~N}=314)=20.549$, $p<0,001, \mathrm{CFI}=0.991, \mathrm{TLI}=0.985, \mathrm{RMSEA}=0.043,90 \%$ IC [.000, .077]; SRMR $=.033$. Las cargas factoriales fueron significativas y con valores fluctuantes entre .485 y .874 ; la correlación entre ambos factores fue de 192 .

Dado que los ítems que se agrupan conformando factores separados son, por un lado, los ítems positivos y los inversos por otro, una posible explicación es que la estructura de la escala estuviese relacionada con la redacción de los ítems, más que conformar dos factores diferentes desde un punto de vista teórico. Sin embargo, ambos factores son interpretables desde un punto de vista teórico y además hay evidencia estadística a favor de una solución de dos factores por sobre una unifactorial. En primer lugar, en el modelo unidimensional con errores correlacionados, los pesos factoriales de los ítems 3, 4 y 6 están por debajo de lo aceptable (entre .096 y .186). En segundo lugar, si bien el ajuste del modelo de un factor con errores correlacionados fue satisfactorio, $\chi 2(11, \mathrm{~N}=314)=16.618, p<0,001$, CFI 
$=0.993, \mathrm{TLI}=0.987, \mathrm{RMSEA}=0.040,90 \%$ IC $[.000$, $.077]$; SRMR $=.018$, la diferencia con un modelo de dos factores, mediante la comparación de modelos anidados no fue significativa, $\Delta \chi^{2}=3.93 \Delta \mathrm{gl}=2, p>0.01$. Considerando estos antecedentes, la solución más adecuada para la Parte B del PAST sería una de dos dimensiones: un primer factor que daría cuenta del acuerdo en el cuidado y educación de los hijos y otro que aludiría a conflictos en el ejercicio de la coparentalidad.

\section{InarianzaMétricadel PAST-A}

Respecto de la comparación entre hombres y mujeres, los resultados presentados en la tabla 3 dan cuenta de la presencia de invarianza configural, métrica y escalar, por lo que la estructura factorial PAST-A es equivalente entre hombres y mujeres.

La evaluación de la invarianza según tipo de relación (ver tabla 4), revela la equivalencia a nivel configural y métrico. A nivel escalar, si bien la comparación de ambos modelos arroja una diferencia significativa $\Delta \chi^{2}, \Delta \mathrm{gl}=18, p$ $=0,007$, las diferencias en los índices de ajuste incremental están por debajo de los criterios establecidos, por lo que puede sostenerse que la estructura delPAST-A es equivalente según tipo de relación.
En síntesis, los ítems cargan en los mismos factores en cada grupo, los pesos factoriales son de similar magnitud y los interceptos son iguales entre los grupos. Es decir, el PAST-A es equivalente por género y tipo de relación.

\section{Consistencia Internadel PAST}

Los análisis de consistencia interna de las subescalas del PAST mediante el alfa ordinal de Omega dan cuenta de valores adecuados, obteniéndose .93 para la subescala de apego a la ex pareja y .94 para la de soledad negativa. Respecto de la escala de coparentalidad, se obtuvo un Omega de .91 para la dimensión de acuerdo y cooperación y .80 para la de conflicto parental.

\section{Validez Concurrente del PAST}

A fin de examinar la asociación delPAST con variables afines pero diferentes, se estimaron los índices de correlación, que se presentan en la Tabla 5. De acuerdo a lo hipotetizado, ambas dimensiones del PAST-A se asociaron a los indicadores de salud mental y a la satisfacción con la vida.

Específicamente, la soledad negativa y el apego a la ex pareja se asociaron positivamente con síntomas de depresión, ansiedad y estrés y negativamente con la satisfacción

Tabla 3. Índices de ajuste para Invarianza Métrica del PAST-A según sexo

\begin{tabular}{lccccccccc}
\hline & $\chi^{2}$ & $\mathrm{gl}$ & $\mathrm{CFI}$ & RMSEA & $\Delta \chi^{2}$ & $\Delta \mathrm{gl}$ & $\Delta$ valor-p & $\Delta \mathrm{CFI}$ & $\Delta$ RMSEA \\
\hline Invarianza Configural & 420.975 & 176 & .951 & .068 & & & & & \\
Invarianza Métrica & 435.576 & 189 & .950 & .066 & 14.601 & 13 & .333 & .001 & .002 \\
Invarianza Escalar & 449.664 & 202 & .950 & .064 & 14.088 & 12 & .325 & .000 & .002 \\
\hline
\end{tabular}

Nota: Mujeres $=344 ;$ Hombres $=250$

Tabla 4. Índices de Ajuste para Invarianza Métrica del PAST-A según Tipo de Relación

\begin{tabular}{lccccccccc}
\hline & $\chi^{2}$ & $\mathrm{gl}$ & $\mathrm{CFI}$ & RMSEA & $\Delta \chi^{2}$ & $\Delta \mathrm{gl}$ & $\Delta$ valor-p & $\Delta$ CFI & $\Delta$ RMSEA \\
\hline Invarianza Configural & 470.360 & 176 & .942 & .075 & & & & & \\
Invarianza Métrica & 485.520 & 189 & .941 & .073 & 15.160 & 13 & .300 & .001 & .002 \\
Invarianza Escalar & 510.130 & 202 & .939 & .072 & 14.088 & 12 & .041 & .002 & .001 \\
\hline
\end{tabular}

Nota. Matrimonio $=326 ;$ Convivencia $=268$ 
Tabla 5. Medias, desviaciones estándar y correlaciones entre las dimensiones del PAST e indicadores de Salud Mental y Satisfacción con la vida

\begin{tabular}{lccccccccc}
\hline Variables & $\mathrm{M}$ & $\mathrm{DE}$ & 1 & 2 & 3 & 4 & 5 & 6 & 7 \\
\hline 1. Soledad negativa & 14.22 & 6.71 & - & & & & & & \\
2. Apego a la ex pareja & 12.37 & 6.32 & $.690^{* *}$ & & & & & & \\
3. Cooperación parental & 9.00 & 4.35 & .013 & .044 & & & & & \\
4. Conflicto parental & 8.04 & 3.66 & .013 & .033 & $.363^{* *}$ & & & & \\
5. Depresión & 3.81 & 4.72 & $.595^{* *}$ & $.437^{* *}$ & .005 & .015 & & & \\
6. Ansiedad & 2.97 & 4.13 & $.457^{* *}$ & $.318^{* *}$ & .034 & -.018 & $.785^{* *}$ & & \\
7. Estrés & 4.65 & 4.59 & $.507^{* *}$ & $.317^{* *}$ & .031 & .068 & $.795^{* *}$ & $.779^{* *}$ \\
8. Satisfacción con la vida & 4.91 & 1.06 & $-.354^{* *}$ & $-.300^{* *}$ & .161 & $-.171^{*}$ & $-.328^{* *}$ & $-.201^{* *}$ & $.240^{* *}$ \\
\hline
\end{tabular}

Nota: $* p<.05 ; * * p<.01$

con la vida en magnitudes leves a moderadas ( $\mathrm{r}=.19$ a .595$)$. Sólo se identificó una correlación inversa de baja magnitud del PAST-B con la satisfacción con la vida.

\section{Discusión}

El objetivo de este estudio fue evaluar la validez y confiabilidad de las puntuaciones de la escala PAST en una muestra de personas separadas y divorciadas chilenas. Los análisis dan cuenta que el instrumento posee, en términos generales, características y propiedades psicométricas adecuadas para su aplicación en dicho contexto.

Los análisis de la Parte A de un modelo de dos factores con errores correlacionados evidenció un adecuado ajuste a loa datos, siendo similar a lo reportado por Sweeper \& Halford (2006) y Lamela et al. (2014) en la validación en una muestra portuguesa. Al igual que en esta última investigación, hubo dos ítems que en nuestra muestra no funcionaron adecuadamente (ítems 6 y 14) y otros dos específicos de este estudio. De este modo, la versión chilena del PAST-A quedaría constituida por 15 ítems.

En relación con la Parte B, orientada a la evaluación de la coparentalidad, los resultados obtenidos en nuestra muestra dan cuenta de una estructura de dos factores, a diferencia de lo propuesto por Sweeper \& Halford (2006), que identificaron una solución unidimensional. Este hallazgo es coherente con estudios que indican que la coparentalidad sería un constructo más bien multidimensional (Lamela \& Figuereido, 2016; Teubert \& Pinquart, 2011). En este caso, uno de los factores correspondería al acuerdo respecto del cuidado y educación de los hijos y el otro se referiría al conflicto parental, siendo concordantes con dos de las dimensiones propuestas por Teubert \& Pinquart $(2010,2011)$.

Adicionalmente, se identificó que la estructura factorial del PAST-A es invariante según género y tipo de relación (matrimonios y uniones de hecho), aportando con ello información adicional sobre este instrumento, dado que la equivalencia del PAST-A entre hombres y mujeres, así como entre personas provenientes de matrimonios y uniones de hecho no había sido testeada previamente en ninguno de los estudios disponibles revisados. De esta manera, es posible sostener que las posibles comparaciones entre dichos grupos son válidas, dado que las puntuaciones del PAST-A tienen el mismo significado entre hombres y mujeres por un lado y entre personas provenientes de matrimonios y uniones de hecho por otro.

Los indicadores de consistencia interna del PAST fueron satisfactorios, siendo la soledad negativa la subescala con mayor fiabilidad, con valores similares a los reportados por Sweeper \& Halford (2006), Lamela et al. (2014) y Rodríguez (2011).

Por otra parte, y en línea con lo reportado en investigaciones previas, identificamos asociaciones moderadas entre las dimensiones de soledad negativa y apego a la ex pareja e indicadores de salud mental y bienestar. Específicamente, la soledad negativa y el apego a la ex pareja se asociaron a mayores índices de depresión, ansiedad y estrés, así como a menores niveles de satisfacción con la vida (Forste \& Heaton, 2004; Sweeper \& Halford, 2006). No se identificó asociación entre los aspectos de la coparentalidad y los indicadores de salud mental, aunque sí una relación inversa pero de baja magnitud entre el conflicto parental y la satisfacción con la vida. Este resultado es en cierto modo 
esperable, dado que a diferencia de lo que ocurre con la subescalas del PAST-A, referidas a la percepción de cómo se vive internamente el proceso de separación, las preguntas sobre coparentalidad evalúan la percepción de una dinámica relacional (versus individual) entre la persona respondiente, el/la ex pareja y los hijos. Los estudios sobre este tema documentan más bien un efecto de las características de la coparentalidad sobre la salud mental de los hijos (Lamela $\&$ Figuereido, 2016) que sobre los padres.

Las limitaciones del estudio incluyen la necesidad de tener cautela respecto de la generalización de los resultados a personas con menor nivel de escolaridad, debido a que la muestra de este estudio estuvo compuesta principalmente por personas con estudios superiores. La misma precaución se debe de tener con los resultados de padres con hijos menores de 18 años, debido a que la muestra que respondió la escala de coparentalidad fue reducida y, por ende, no pudieron llevarse a cabo estudios de invarianza según género y tipo de relación. Futuros estudios podrían subsanar estas limitaciones evaluando el comportamiento particularmente de la PAST en una muestra más amplia para la parte B y con características más heterogéneas.

Desde un punto de vista clínico, consideramos que contar con un instrumento de este tipo en el contexto chileno tiene utilidad para el trabajo con personas y familias en proceso de separación, pues otorga una herramienta para evaluar cómo se encuentran las personas que solicitan ayuda psicoterapéutica en aspectos específicos de ajuste al divorcio y no sólo indicadores generales sobre su salud mental, que pueden tener o no relación con la ruptura misma. Disponer de instrumentos de este tipo posibilita además contar con una herramienta para el diseño y medición del impacto de intervenciones focalizadas en aquellos ámbitos en que la persona presenta mayores dificultades.

Finalmente y a modo de conclusión, elPAST representa el inicio de un cuerpo de investigación en el ámbito del divorcio y/o separación en Chile, desde la perspectiva de la psicología clínica, dado que los estudios disponibles a la fecha han sido abordados desde una mirada predominantemente jurídica (Cox, 2011) y/o sociológica (Gómez, 2012).

\section{Referencias}

Afifi, T. O., Cox, B. J., \& Enns, M. W. (2006). Mental health profiles among married, never-married, and separated/divorced mothers in a nationally representative sample. Society Psychiat Epidemiol, 41, 122-129. doi:10.1007/s00127-005-0005-3

Amato, P. (2000). The consequences of divorce for adults and children. Journal of Marriage and the Family, 62, 1269-1287. doi:10.1111 /j.1741-3737.2000.01269
Amato, P. R. (2010). Research on divorce: Continuing trends and new developments. Journal of Marriage and Family, 72, 650-666. doi:10.1111/j.1741-3737.2010.00723.x

Bentler, P. M. (1990). Comparative fit indexes in structural models. Psychological Bulletin, 107, 238-246. doi:10.1037/0033-2909.107.2.238

Cáceres C., Manhey C. \& Raies A. (2004). Comprensión sistémico-relacional del proceso de separación conyugal. Revista De Familias y Terapias, 12(18), 31-54

Carr, A. (2009). The effectiveness of family therapy and systemic interventions for adult-focused problems. Journal of Family Therapy, 31, 46-74. doi:10.1111/j.1467-6427.2008.00452.x

Chen, F. F. (2007). Sensitivity of goodness of fit indexes to lack of measurementinvariance. Structural Equation Modeling-aMultidisciplinary Journal, 14, 464-504. doi:10.1080/10705510701301834

Cox, L. (2011). Divorcio en Chile Un análisis preliminar tras la nueva ley de matrimonio civil. Estudios Públicos, 123, 95-187.

Diener, E., Emmons, R., Larsen, R., \& Griffin, S. (1985). The Satisfaction with Life Scale. Journal of Personality Assessment, 49, 71-75. doi:10.1207/s15327752jpa4901_13

Evans, M., \& Kelley, J. (2004). Effect of family structure on life satisfaction: Australian evidence. Social Indicators Research, 69, 303-349. doi:10.1007/s11205-004-5578-9

Eysenck, S., Eysenck, H., \& Barrett, P. (1985). A revised version of the psychoticism scale. Personality and Individual Differences, 6, 21-29. doi:10.1016/0191-8869(85)90026-1

Fisher, B. (1978). Facilitator's manual for rebuilding: When your realationship ends. Boulder, Colorado: Family Relations Learning Center.

Fisher, B. (1981). Aprender a amar de nuevo. Cómo rehacer tu vida cuando una relación termina. México, D.F.: Pax México.

Forste, R., \& Heaton, T. (2004). The divorce generation: Well-being, family attitudes, and socioeconomic consequences of marital disruption. Journal of Divorce and Remarriage, 41, 95-114. doi:10.1300/J087v41n01_06

Gähler, M. (2006). “To Divorce Is to Die a Bit...”: A longitudinal study of marital disruption and psychological distress among Swedish women and men. The Family Journal, 14(4), 372-382. doi:10.1177/1066480706290145 Gómez, V. (2012). Divórcio à la chilena: familia, gênero e cidadania no Chile, 1990-2004. Revista de Sociología e Política, 20, 177-195. doi:10.1590/s0104-44782012000400013

Guzmán, M., Garrido, L., Calderón, C., Contreras, P., \& Rivera, D. (2017). Chilean adaptation and validation of the Fisher Divorce Adjustment Scale-Short Form. Journal of Divorce and Remarriage,

Hambleton, R. K., Merenda, P. F., \& Spielberg, C. D. (2005). Adapting educational and psychological test for cross-cultural assessment. Mahwah, NJ: Lawrence Erlbaum.

Horn, J. L. (1965). On the use of random variables in factor analysis. British Journal of Mathematical and Statistical Psychology, 19, 127129. doi: 10.1111/j.2044-8317.1966.tb00361.x

Hughes, M. E., \& Waite, L. J. (2009). Marital biography and health at mid-life. Journal of Health and Social Behavior, 50, 344-358. doi:10.1177/002214650905000307 
Jakoby, N. (2012). Grief as a Social Emotion: Theoretical Perspectives. Death Studies, 36, 679-711. doi:10.1080/07481187.2011.584013

Kellas, J. K., \& Manusov, V. (2003). What's a story? The relationship between narrative completeness and adjustment to relationship dissolution. Journal of Social and Personal Relationship, 20, 285-307. doi:10.1177/0265407503020003002

Lamela, D., \& Figuereido, B. (2016). Coparenting after marital dissolution and children's mental health: A systematic review. Jornal de Pediatría, 92, 331-342. doi: 10.1016/j.jped.2015.09.011

Lamela, D., Figueiredo, B., Bustos, A., \& Martins, H. (2014). Psychometric Properties of the Portuguese Version of the Posttraumatic Growth Inventory Short Form Among Divorced Adults. European Journal of Psychological Assessment, 30, 3-14. doi:10.1027/1015-5759/a000161

Lorenzo-Seva, U., \& Ferrando, P. J. (2006). FACTOR: A computer program to fit the exploratory factor analysis model. Behavioral Research Methods, Instruments and Computers, 38, 88-91. doi.org/10.3758/ bf03192753

Lovibond, P., \& Lovibond, S. (1995). The structure of negative emotional states: Comparison of the Depression Anxiety Stress Scales (DASS) with the Beck depression and anxiety inventories. Behaviour Research and Therapy, 33, 335-343. doi:10.1016/0005-7967(94) 00075-u

Millsap, R. E., \& Cham, H. (2012). Investigating factorial invariance in longitudinal research. En B. Laursen, T. D. Little \& N. A. Card (Eds.), Handbook of developmental research methods (pp. 109-126). New York, NY: Guilford Press.

Muthén, L. K., \& Muthén, B. O. (2014). Mplus user's guide (7th ed.). Los Angeles, CA: Muthén y Muthén

Pavot, W., \& Diener, E. (1993). Review of the Satisfaction With Life Scale. Psychological Assessment, 5, 164-172. doi:10.1037/1040-3590.5.2.164

Rodríguez, R. (2011). Apoyo social y ajuste psicosocial post divorcio en adultos de Monterrey, México: Explorando el papel del tiempo, fuentes y tipos de apoyo (Tesis doctoral). Universidad Autónoma de nuevo León, Monterrey, México.

Rodríguez, R. \& Ribeiro, M. (2013). Predictores del ajuste psicosocial en divorciados en Monterrey, México: Un análisis de género. Noesis. Revista de ciencias sociales y humanidades, 22, 268-288. Recuperado desde http://www.redalyc.org/html/859/85927874020/

Sbarra, D. A. (2006). Predicting the onset of emotional recovery following nonmarital relationship dissolution: Survival analyses of sadness and anger. Personality and Social Psychology Bulletin, 32, 298-312. doi:10.1177/0146167205280913

Sbarra, D. A. (2015). Divorce and Health. Psychosomatic Medicine, 77, 227-236. doi:10.1097/psy.0000000000000168

Smet, O., Buysse, A., \& Brondeel, R. (2011). Effect of the breakup context on unwanted pursuit behavior. Journal of Forensic Sciences, 56, 934-941. doi: 10.1111/j.1556-4029.2011.01745.

Sweeper, S., \& Halford, K. (2006). Assessing adult adjustment to relationship separation: The Psychological Adjustment to Separa- tion Test (PAST). Journal of Family Psychology, 20(4), 632-640. doi:10.1037/0893-3200.20.4.632
Teubert, D., \& Pinquart, M. (2010). The association between coparenting and child adjustment: A meta-analysis. Parenting: Science and Practice, 10, 286-307. doi:10.1080/15295192.2010.492040

Teubert, D., \& Pinquart, M. (2011). The link between coparenting, parenting, and adolescent life satisfaction. Family Science, 2, 221-229. doi:10.1080/19424620.2012.666655

Vera-Villarroel, P., Urzúa, M. A., Pavez, P., Celis-Atenas, K. \& Silva, J. (2012). Evaluation of Subjective Well-being: Analysis of the Satisfaction With Life Scale in Chilean Population. Universitas Psychologica, 11, 719-727. Recuperado desde http://recursostic.javeriana.edu.co/sitio/ psychologica/sccs/articulo.php?id=3185

Vinet, E., Rehbein, L., Román F., \& Saiz, J. (2008). Escalas abreviadas de depresión, ansiedad y estrés (DASS-21). Versión chilena traducida y adaptada. Documento no publicado, Universidad de La Frontera, Temuco, Chile.

Wang, H., \& Amato, P. (2000). Predictors of divorce adjustment: Stressors, resources, and definitions. Journal of Marriage and the Family, 62, 655-668. doi:10.1111/j.1741-3737.2000.00655.x

Yárnoz-Yaben, S. (2010). Attachment style and adjustment to di- vorce. The Spanish Journal of Psychology, 13, 210-219. doi:10.1017/ s1138741600003796

Yárnoz, S., \& Comino, P. (2010) El CAD-S, un instrumento para la evaluación de la adaptación al divorcio-separación. Psicothema, 22, 157-162. Recuperado desde http://www.psicothema.com/english/ psicothema.asp?id=3711

Yilmaz, A. E., \& Fisiloglu, H. (2006). Psychometric properties of the Fisher Divorce Adjustment Scale in a turkish divorced sample. Journal of Divorce y Remarriage, 45, 149-169. doi:10.1300/J087v45n01_08 